\title{
Requirement of artificial intelligence technology awareness for thoracic surgeons
}

\author{
Anshuman Darbari ${ }^{*}$ (D), Krishan Kumar $^{2}$ (D), Shubhankar Darbari ${ }^{3}$ (B) and Prashant L. Patil ${ }^{1}$ (B)
}

\begin{abstract}
Background: We have recently witnessed incredible interest in computer-based, internet web-dependent mechanisms and artificial intelligence (Al)-dependent technique emergence in our day-to-day lives. In the recent era of COVID-19 pandemic, this nonhuman, machine-based technology has gained a lot of momentum.

Main body of the abstract: The supercomputers and robotics with Al technology have shown the potential to equal or even surpass human experts' accuracy in some tasks in the future. Artificial intelligence (Al) is prompting massive data interweaving with elements from many digital sources such as medical imaging sorting, electronic health records, and transforming healthcare delivery. But in thoracic surgical and our counterpart pulmonary medical field, Al's main applications are still for interpretation of thoracic imaging, lung histopathological slide evaluation, physiological data interpretation, and biosignal testing only. The query arises whether Al-enabled technology-based or autonomous robots could ever do or provide better thoracic surgical procedures than current surgeons but it seems like an impossibility now.
\end{abstract}

Short conclusion: This review article aims to provide information pertinent to the use of Al to thoracic surgical specialists. In this review article, we described Al and related terminologies, current utilisation, challenges, potential, and current need for awareness of this technology.

Keywords: Artificial intelligence, Deep learning, Machine learning, Thoracic surgery, Robotic surgery, Thoracic cancers

\section{Background}

Scientific advances from multiple disciplines have already played a significant role in every branch of medicine, including thoracic surgery. The researches and techniques derived from more fields are usually required to solve problems and advancements. It is the rapid development of physics, chemistry, and biochemistry supported by mathematics, which has made various medical advances possible: Whether it is the development of optical physics for microscope and telescope, the discovery and use of radiation physics, or the advancements in the field of clinical laboratory and pathology investigations.

\footnotetext{
* Correspondence: darbarianshu@gmail.com

${ }^{1}$ CTVS Department, AllMS, Rishikesh 249203, India

Full list of author information is available at the end of the article
}

Therefore, every thoracic surgeon must have a basic understanding of the fundamental science with recent advances to obtain a multi-dimensional progressive view of the field. New scientific advancements will help thoracic surgeons to expand an existing skillset and perform new innovative procedures.

While it is a known fact that each generation of physicians significantly exceeds the previous generation's accomplishments based on previous discoveries. Nonetheless, modern science is accelerating quicker than ever due to the advent of computer science, especially artificial intelligence (AI) development, and it seems we are on the edge of another revolution. Future surgeons will need to know and grasp a broader range of 
the modern technology advancements quicker than ever before [1].

The Oxford Dictionary defines "AI" as the development of computer machine systems to accomplish tasks requiring human brain intelligence such as visual perception, speech recognition, decision-making, and language translation [2]. AI-based technologies mainly focus on the machine which allows the computers to learn from data and interpret, based on a predefined algorithm and reproduce near-human brain interpretations. The networks in AI systems are designed to mimic the same way as human brain processes information and derive solutions. Multiple of these neural networks with base algorithms work together to get a final decision. AI-based technologies are already prevalent in many areas of our daily life. Google Maps uses AI to pick up traffic patterns and create efficient routes dynamically, and smartphones use AI to recognise facial and voice-based commands for routine daily tasks such as internet data searches and speech recognitions. But in the healthcare industry, its extensive use is still limited and started recently. Thoracic surgeons should be aware of this technological advancement and recent development that could augment their clinical practice.

The MEDLINE (Ovid) and PubMed databases were searched for articles and papers (Books and Documents, Clinical Trial, Meta-Analysis, Randomized Controlled Trial, Review, Systematic Review) for the last 5 years that included keywords from both of two categories: thoracic surgery and artificial intelligence (keywords: artificial intelligence, machine learning, neural network, computer-assisted surgery) with search filters applied as above. A total of 41 publications were found, and further from this list, articles related to cardiac surgery, echocardiography, mitral valve repair, and trans-catheter cardiac treatment modalities were excluded. The exact best match was found for 3 articles only. We present the following article in accordance with the journal's review reporting checklist.

\section{Main text}

\section{Basics of technology/terminology}

The field of AI was started at Dartmouth College (Hanover, NH, USA) in 1956, where a group of computer scientists assembled to discuss mathematical theorems, language processing, game theory and how computers learn from analysing training examples [3]. At first, a rules-based system governed AI. But by the 1980s, medical uses were noted and experimented. AI is a mixture of computer science and robotics, but it focuses more on empirically processing machine behaviour with output. Essentially, the human programmer defined what the computer has to do. Now, AI-based technologies uses adaptive analytical power with autonomous learning and complex algorithms. Algorithms that could represent medical knowledge are coded into the system as data and provide direction for different clinical scenarios. Pattern recognition also allows AI to separate anatomical structures for visualisation and analysis. So, now AI-based systems can recognise patterns, anticipate future events, make sound decisions, learn and unlearn from mistakes, assist clinical decision-making, and discover relevant information from data.

Machine learning (ML) is a subfield of AI in which statistical models are used to learn patterns from data to accomplish a specific task. With ML, modern systems are considered more intelligent because they use algorithms that enable the machine (or Computer) to learn functions from one particular and potentially everchanging dataset. ML algorithms can be divided into three types: supervised learning, unsupervised learning, and reinforcement learning. ML techniques range from simple linear models such as logistic regression and naïve Bayes classifiers to complex neural network models with many parameters. In this, the computer programme can somewhat learn by itself, and software can automatically adapt its behaviour to match that specific task's requirements [4].

Deep learning (DL) is a more inner subfield of the AI branch in which features needed for particular task completion are automatically learned from the raw data. But, DL requires costly and time-consuming human effort to accumulate raw data, provide statistical analysis and domain expertise, and build prior experimental models with specific feature sets and potentially complex algorithms. DL often overtakes other ML modalities when these massive datasets are manipulated. The development of DL is closely related to neural networks. It usually relies on principles similar to the human brain's working, composed of input and output layers of data representations called neurons. There are also various buried layers (neurons that cannot be defined in any specific either input or output layers). All layers are arranged sequentially so that a representation of one layer is fed into the following layer, the depth of the network being linked to the number of hidden and output layers. In more complex and deep networks, more powerful models to learn complex nonlinear mappings can be developed. These deep learning networks are trained by the method of 'iteration', which is a process of running and re-running networks with continuous optimising neuronal parameters to improve performance and minimise errors. Massive datasets drive this technological improvement with a pursuit for better algorithms and faster computational hardware. Figure 1 is the compiled representation of the various definitions in the AI field. 


\section{Artificial Intelligence}

The technology is to proceed on the mechanical basis of estimation that every aspect of leaning or human intelligence can so precisely described that a machine can be made to simulate it.
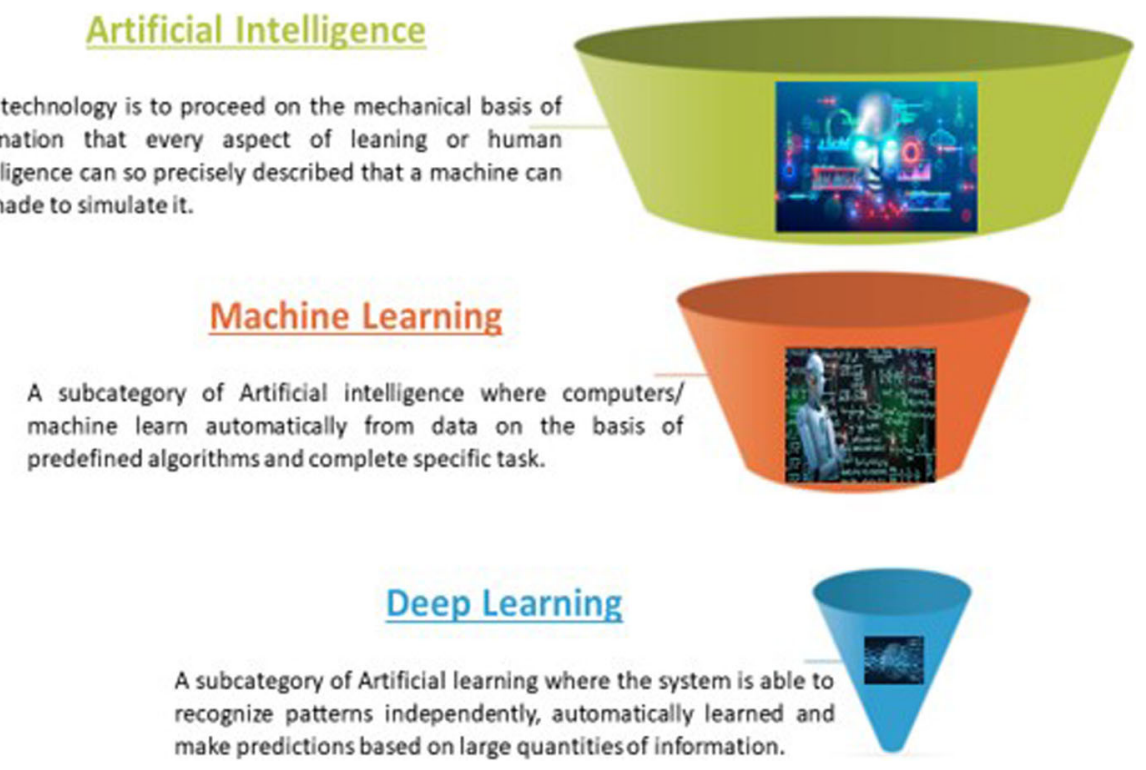

Fig. 1 Various fields of artificial intelligence and definitions

The explosion of interest in medical applications of AI and mainly DL during recent years may also be attributed to the convergence of especially two key factors:

1. Big data analysis and faster computation

2. Artificial neural networks (ANNs) or deep neural networks (DNNs): these networks are loosely modelled on the human brain mechanism, and consist of multiple layers of 'neurons' that successively process input data until the output layer is reached. DNNs are a recently developed variant of ANNs that have a large number of intermediate buried layers (often more significant than 10) and process input data hierarchically, with the first few layers responding to simple low-level features (such as straight lines) and successive layers responding to more abstract high-level features (such as the shape of specific objects) [5-7].

In recent years, with faster computer equipment and radiomics, AI has already made significant progress in medical imaging evaluation and radiological diagnosis of

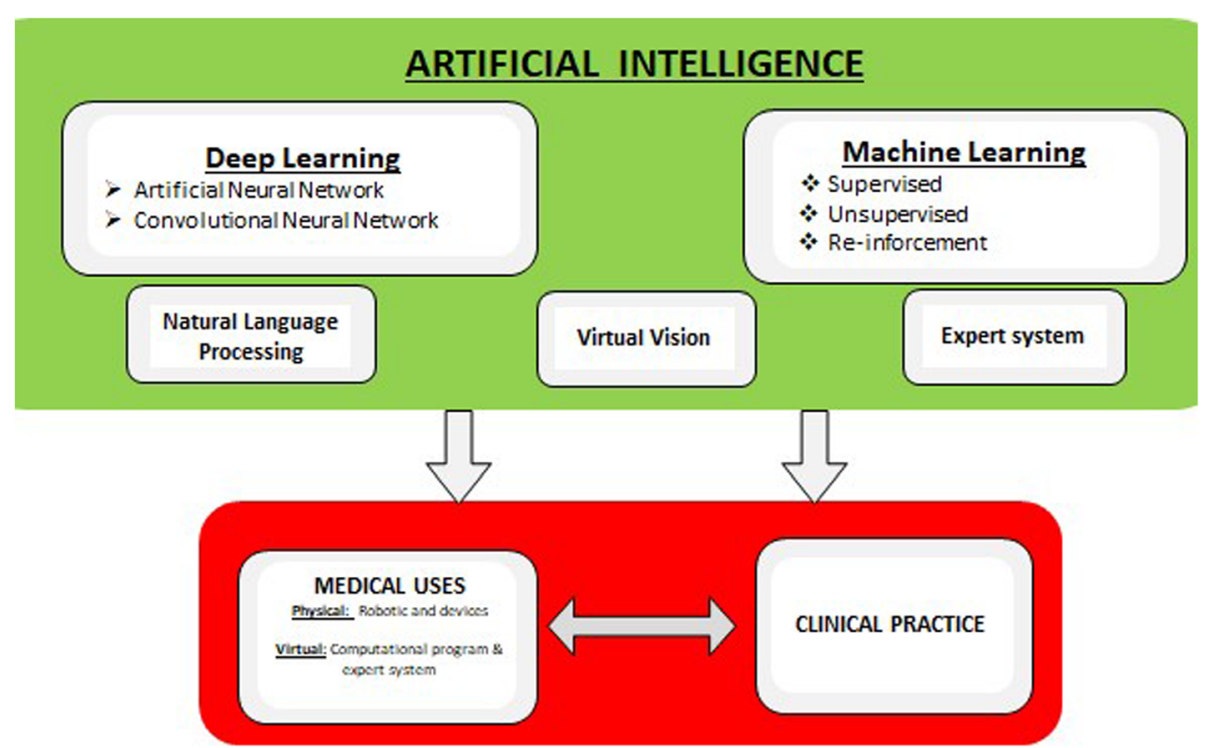

Fig. 2 Components of artificial intelligence and use in the medical field 
pulmonary diseases. Radiomics typically consists of five components: data selection, medical imaging, feature extraction, exploratory analysis, and modelling. Radiomics consist of extracting and analysing imaging features such as shape, texture or intensity of voxels from radiographic medical images using data-characterisation algorithms and relies on statistics. Radiomics is currently very much in use by pulmonary radiologists [8]. Figure 2 is a schematic representation of the various AI domains and interplay for medical usages.

\section{Current utilities}

Initially, the AI performance and involvement was limited by low sensitivity, incomplete medical knowledge datasets and insufficient ability to integrate probabilistic reasoning. The chronological evolution history of AI and usage in the medical field is congregated in Table 1 [9]. The advances that have been already executed with the help of AI and DL are now well-designed in various aspects of pulmonary medicine but with limited usefulness in thoracic surgery. The significant medical field advancements that have been developed with the help of AI technique may be broadly categorised under these headings for thoracic surgical specialists:

1. Thoracic imaging interpretation and diagnosis making.

2. Histopathology or cytology assessment to aid in proper diagnosis.

3. Physiological measurements and bio-signal testing.

4. Virtual reality platforms and preoperative AI-based planning for lung resections. AI-based medical brain

Table 1 Timeline of major development in Al field with medical applications

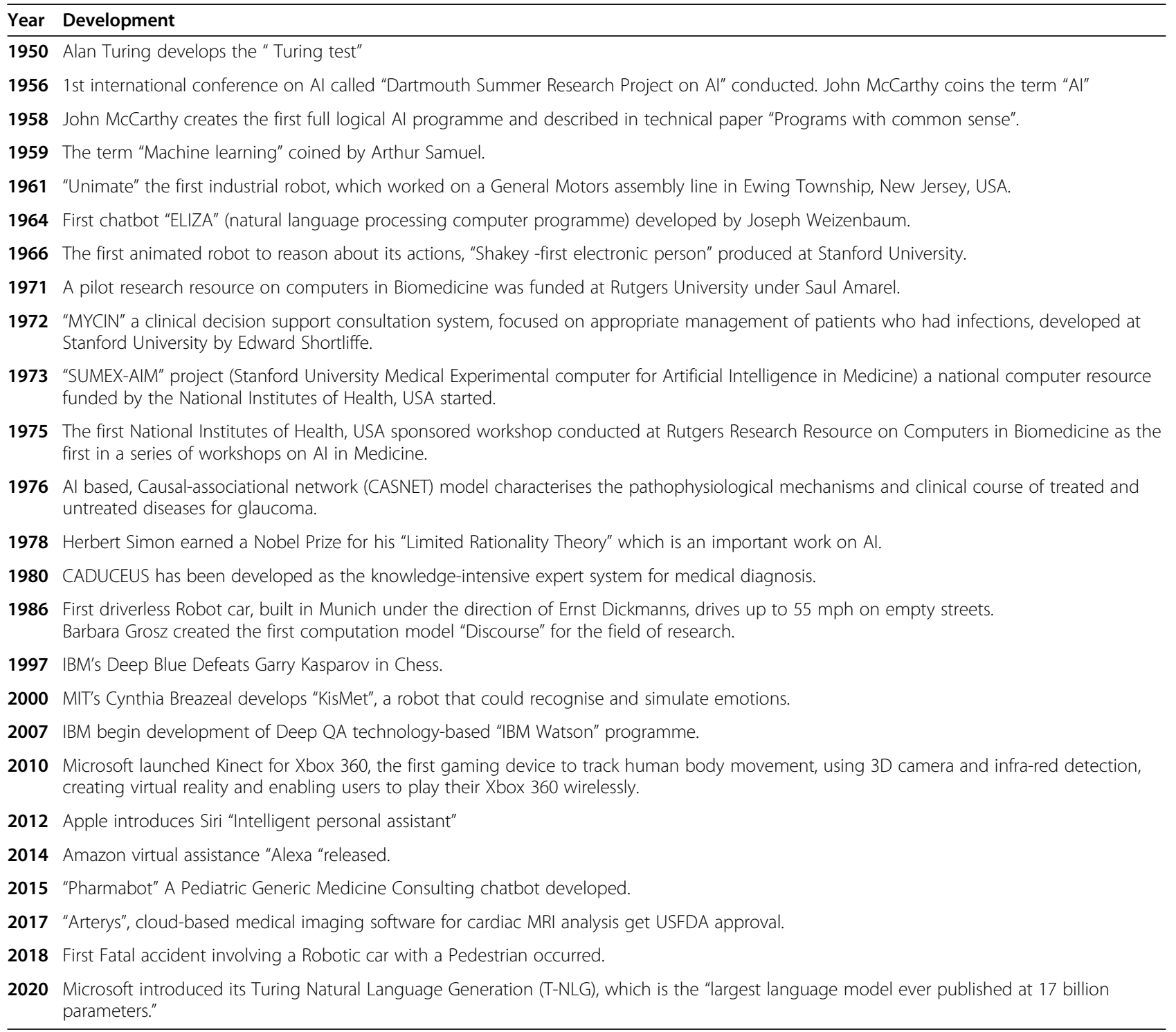


servers to interpret patient data and diagnosis making.

5. Robotic / Machine assisted surgery, including automatic camera handling devices, Robotic surgery platforms, and voice-controlled robotics.

6. Intraoperative usage of AI-based endoscopic systems for identification of tumours during surgery and virtual endoscopy.

7. AI-based algorithms for overall risk assessment and risk scoring with prognostication.

8. Surgical education and training.

AI has been initially used in the diagnosis of lung nodules by use of Radiomics. Computer-aided diagnostics algorithms were developed initially as an assistance tool for radiologists to detect chest radiograph lesions. These algorithms were subsequently used for similar purposes for computed tomography (CT), magnetic resonance imaging (MRI) image interpretations, and the nuclear medicine field. These AI-based algorithms provide not only second opinions to assist radiologists but also save precious time. While the heavy workload of several chest $\mathrm{x}$-rays or Chest CT films examination by radiologists for detecting precisely lung nodules or masses can be challenging tasks, AI-based algorithms and software can serve as valuable diagnostic aids, especially for suspicious lung nodules. By automating these simple tasks, radiologists are freed, and it demonstrates an improvement in human capital use by clinicians and synergistic cooperation with AI technology. In 2011, National Lung Screening Trial (NLST) for early detection of lung cancer by low-dose CT scan and in 2018, Netherlands Leuvens Longkanker Screening ONderzoek (NELSON) trials had provided an extensive data set to explore using AI. The NLST found a relative reduction in lung cancer mortality with low-dose CT screening of $20 \%$ with a $95 \%$ confidence interval (CI). The Nelson study is another randomised lung cancer screening trial using low dose CT that showed very promising results and is more favourable than the NLST results but suggests gender differences. Ardila et al. developed an alternative deep convolutional neural network for a complete assessment of low-dose CT volume focusing on concern regions of concern compared to prior imaging when available and standardisation against biopsy-confirmed outcomes [10]. Haitao Niu recently described the very promising experimental results of a visual feature extraction model based on AI with thoracic echo spectrum, and they established the effectiveness of this method compared with the latest approaches for radiological diagnosis of lung cancers [11].

We are also witnessing the recent development of various AI-based applications of digital pathology assessment for histopathological diagnosis. By researching the large archival databases of digital pathology images and detailed image analysis facilitate accurate diagnosis. It can also provide support from remote institutes for an expert second opinion [12].

Topalovic et al. have already demonstrated that AIbased algorithms might outperform the pulmonologists to interpret pulmonary function tests. AI-based auscultatory lung sound analysis of normal and adventitious lung sounds has been tried, but as these sounds are highly variable, henceforth, it is a challenge in generalising these algorithms based on decisions [13].

Most recently, Sadeghi et al. have presented the first pilot study about the development of an immersive virtual reality-based platform (PulmoVR) that enables personalised surgical planning for pulmonary segmentectomies. This is the first dedicated virtual reality-based segmentectomy planning tool that provides accurate surgical planning. But, this study contains only ten patients operated by two thoracic surgeons, so future clinical studies can further evaluate and demonstrate the clinical benefits of such technology. This is the classical procedure of using virtual components of AI practice in the preoperative planning field [14]. Previously, Yang et al. also described image-based software use for locating the nodules with the 3D images and coloured segments merger. The clearly labelled lung structure was utilised in a preoperative virtual segmentectomy and the subsequently planned thoracoscopic surgery [15].

In contradiction of the medical field, the thoracic surgical field has near negligible use of AI-based technologies, especially for benign disease management. As described above, in thoracic oncology patient management, the virtual component of $\mathrm{AI}$ is being used. In pure terms, surgeons are using the mechatronic or robotic branch of AI in a very limited manner and the term 'surgical robots' are often mistakenly interpreted in the AI context [16]. Although the term 'robotic surgery' is often applied to the da Vinci surgical system device-assisted surgery, it is a misconception of fully autonomous surgery since it cannot be self-programmed, nor can it make independent decisions to perform any surgical procedure, and characteristically, it is only a masterslave kind of assist device. In the exact terminology, current robotic devices are only assistance device with an autonomy of level-2 (on a scale of level 1 to 5 in the robotic science field). Other AI physical componentbased, assistance devices are in use such as robotised endoscope positioner for a stable view of the operating field with the elimination of camera shake by a human assistant. It makes thoracoscopic procedures more ergonomic with less physical discomfort for the surgeon by saving movements and enhanced precision [17]. Another experimental development in the robotic surgical field started in 2005 is voice-controlled and lightweight; low 
cost parallel robot PARAMIS (PARAllel Robot for Minimally Invasive Surgery (MIS) in Centre for Industrial Robots Simulation and Testing (CESTER) within the Technical University of Cluj-Napoca, Romania by Prof. Doina Pisla and team. These are initial steps toward a multi-arm, low-cost robot with other additional modalities, which can be used in experimental laparoscopic surgery. The first porcine cholecystectomy has been successfully performed and reported by this team. The experimental results recommend PARAMIS as a potentially valuable tool for MIS. The cost of such a robot is only about 20,000 Euro, which is very cheap compared to the other commercial laparoscope holder devices or available assisting robots. But, this is still an experimental prototype model without any human use approval $[18,19]$. In 2016, Shademan et al. used smart tissue autonomous robot (STAR) and compared various metrics of intestinal anastomosis in pigs (consistency of the suturing, the pressure at which anastomosis leaked, the number of mistakes or removing the needle from tissue, completion time and lumen reduction in intestinal anastomoses) between a supervised autonomous system STAR, manual laparoscopic surgery, and clinically used robotic-assisted surgery in pig experiments. The supervised autonomy with STAR surpassed robotic-assisted surgery, laparoscopic approach, and manual surgery based on some metrics. The interactive decision-making and execution between the surgeon and the STAR system made way for a new exciting and innovative possibility to augment surgeons. This study demonstrated that in future, this kind of autonomous human surgery is possible. The STAR system established the feasibility of performing pig intestinal suturing. Still, there has been no US Food and Drug Administration (USFDA) approved clinical, surgical application in humans despite further research so far [20, 21]. The development history of approved robotic surgery is summarised in Table 2 [22]. But with the rapid advent of AI in the future, it seems promising that the AI-based robotic system could carry out the operative intervention by itself with full autonomy of level-5 and self-adaptation to different operative scenarios like the upcoming AI-based Tesla fully self-driving autopilot car [23]. Considering this future possibility, the European Union have discussed the possible future circumstances in which a robot can take autonomous decisions. It was debated that by the traditional rules, it would not make it possible to ascertain the party responsible for providing compensation in case of an accident and require the party to make accountable for any damage it has caused. The solution suggested was to consider a definite legal status for robots as 'electronic persons' for making them guilty for any possible damage they may cause [24].

Recently, AI-based technology development in the intraoperative surgical field in clinical trials is also shown for easy identification of tumours and margins with the help of AI-based diagnostic algorithms with in vivo optical imaging for quantitatively analysing images [25].

Santos Gracia et al. evaluated an AI-based artificial neural network's performance for predicting cardiopulmonary morbidity after Lung resection for non-small cell lung cancer (NSCLC). In this study, sensitivity and

Table 2 Robotic surgery development history

\begin{tabular}{|c|c|}
\hline Year & Development \\
\hline 1921 & Czech playwright Karel Capek introduced the notion and coined the term "Robot" in his play Rossom's Universal Robots \\
\hline 1985 & First Robotic assist surgery performed by "PUMA 560" to obtain brain biopsy by Kwoh et al. \\
\hline 1988 & Transurethral prostate resection performed by "PROBOT" at Imperial College London. \\
\hline 1992 & $\begin{array}{l}\text { Integrated Surgical Supplies Ltd. of Sacramento, CA, developed ROBODOC to machine the femur with greater precision in hip replacement } \\
\text { surgeries - first surgical robot approved by the USFDA. }\end{array}$ \\
\hline 1993 & AESOP (made by Computer Motion company) telescope manipulator introduced. \\
\hline 1995 & Intuitive surgical company is founded. \\
\hline 1997 & First laparoscopy procedure is performed by a Robotic system. \\
\hline 2000 & Da Vinci (Intuitive Surgical) and ZEUS (Computer Motion) surgical systems approved by FDA. \\
\hline 2001 & First distant transatlantic robot-assisted procedure (cholecystectomy) done. \\
\hline 2003 & Computer Motion and Intuitive Surgical merge into one company. ZEUS company is discontinued \\
\hline 2004 & "Spine Assist" device by Mazor Robotics Ltd., a miniature robotic guidance system received USFDA approval for spinal procedures. \\
\hline 2007 & "Sensei-X" device developed by Hansen Medical company received USFDA approval for intravascular procedures. \\
\hline 2012 & 23,000 hip and knee procedures conducted globally with "MAKO" robotic system by The Stryker company. \\
\hline 2013 & As reported, Over 500,000 surgical procedures performed worldwide with the da Vinci system. \\
\hline 2016 & In the USA, surgical figures came out that over half of prostatectomies and a third of hysterectomies are performed robotically. \\
\hline 2019 & Over 5000 da Vinci surgical systems installed worldwide and approximately 6 million surgeries conducted globally. \\
\hline
\end{tabular}


specificity for predictions were 0.67 (95\% CI $0.49-0.79$ ) and 1.00 (95\% CI 0.97-1.00), respectively. Similarly, Esteva et al. used four different probabilistic Ann's models, trained by data from 113 patients of NSCLC. As we all know, prognosis plays an essential role in patient management in thoracic oncology surgical practice because it implies the efficacy of the surgery with an overall impact on the patient's short- and long-term outcomes. AI-based systems have also shown promising results and assistance tools to decision-making for thoracic surgery patients by evaluating their surgical risk and estimating their prognosis [26]. AI-based computer modelling systems are now an established powerful tool for patient-tailored treatment in the pulmonary medicine field.

Another potential use of AI is in surgical education and training by creating virtual reality platforms and different clinical scenarios. This technology can be used for surgical skill assessment and optimising the training of surgeons with an objective evaluation of the surgical trainees [27].

\section{Challenges}

AI and DL usages in medical fields are still very challenging because of difficulties for conventional statistical analysis methods such as logistic regression to isolate and confirm relationships between predictors and outcomes, especially when relationships are nonlinear and with many individual and operators variables. The quality of the AI's output or answers largely depends on the available data's quality and amount. Moreover, most datasets include a large amount of information and various inputs, so the information provided by the data may not allow to get a single best output.

The applicability of various AI algorithms across other medical fields is critically dependent on multiple factors such as exact representativeness of included populations, missing data links, and outliers. For example, any specific thoracic disease that affects any population group in one continent may not be existent with the same epidemiologic characteristics in another continent or even subcontinent area population. Therefore, underrepresentation of a particular group of people may have a risk of selection bias which may impact final generalised predictions. By inputting low socioeconomic status as a significant risk factor for premature mortality, $\mathrm{AI}$ algorithms may also be biassed against patients of a particular ethnicity or socioeconomic status due to incomplete baseline data, which may widen the gap in health outcomes. Embedded prejudice can only be prevented by massive, robust datasets of a true representative cross-section of all populations. Another example relates to the inequalities of healthcare delivery and the hefty financial expenses of these services. Vital Surgical decision making involves the complex analysis of a patient's clinical assessment with physician intuition based on clinical experience while considering all relevant medical history, adjustable risk factors, and possible complications with the patient's emotional expectations. Each decision-making step introduces considerable variability and opportunities for probable errors, especially in critical thoracic surgical condition management.

Nevertheless, external validation by using an independent dataset is of utmost critical before implementation in real-world clinical practice with inherently opaque AI algorithm black-box models. In the current era of evidence-based medicine, clinical decisions are based on the data provided by large-scale randomised clinical trials. Another hurdle of the generous use of AI in healthcare is the lack of randomised and prospective validation studies with difficulty improving an algorithm's performance on a theoretical basis only [28].

One illustration of iatrogenic harm related to AI-based algorithms is already on records by hundreds of hospitals worldwide using the IBM Watson supercomputer system for recommending cancer treatments in 2018. Algorithms based decisions on a small number of artificial theoretical, fictional cases using limited input from oncologists resulted in wrong output, and treatment recommendations. This faulty finding required systemic debugging, exhaustive audits, extensive iteration and evidence from other robust, and prospective validation studies before algorithms are widely implemented in actual clinical practice.

Although their accuracy is impressive, a drawback of $\mathrm{AI}$ and DL is also their lack of interpretability. The features used to distinguish between data categories are not readily translated into verbal or visual rules that any human can easily understand. Inculcating AI into our data process methods and make medical decisions may also take time. Prospective validation studies, particularly in clinical settings, are still lacking, and there is some valid fear that over-reliance on AI-based technologies might result in de-skilling our human workforce. There may be unsatisfactory outcomes, especially if initial algorithms are poorly generalisable or built on unstructured or unreliable data. In addition, due to the lack of technical awareness and its potential usefulness, many healthcare professionals identify AI as a threat to future medical jobs [29].

As of now, recent developments are focused more on the virtual component of $\mathrm{AI}$, considering the human brain as a replica model. In surgical fields, especially thoracic surgery, which also need keen visual perceptions, fine tactile sensation, and delicate hand dexterity with hand-eye coordination as key significant components for successful surgery completion, the physical component of $\mathrm{AI}$ is still lagging behind. 
There is also word of cautions by Notable scientist Stephen Hawking, Microsoft founder Bill Gates, history professor Yuval Noah Harari, and Space-X founder Elon Musk. They have expressed serious concerns about the possibility that AI could evolve to the extreme uncontrollable point, with Stephen Hawking even speculating that this could "spell the end of the human race as once humans develop AI, it will take off on its own and redesign itself at an ever-increasing rate and humans, who are limited by slow biological evolution, couldn't compete and would be superseded".

\section{Future ahead}

AI-based various machineries are already an integral part of our daily lives. As AI encompasses all three modalities and increasing computational power with the massive datasets that make up 'big data', it is also encouraging for healthcare applications. As our ability to manipulate images, language and speech increase with the help of AI and with the advent of new hardware as neuromorphic chips and quantum computing, larger datasets will become easily analysable. Other medical specialities such as radiology and pathology are most receptive to AI-based applications. As of now, more than 75 AIbased algorithms are approved by the USFDA, and AIbased medical imaging investments in the radiology and pulmonary medicine field have grown exponentially to USD 1.17 billion in the last years. To our knowledge, after an extensive literature search, various algorithms related to pulmonary medicine have also received premarket approval from USFDA recently in the previous two years, and several more are CE-marked (mandatory regulatory marking for products within the European Union economic area). Lagging growth in the thoracic surgery field compared with some other medical fields also warrants a need for increased responsiveness among doctors of these specialities for this advancement [30].

Several commercial establishments and academic institutions are in the phase of building massive clinical datasets with the hope that DL derived algorithms will favourably impact disease prediction, prevention, diagnosis, treatment and boost in healthcare-related economics. The recent development of medical brain platforms by AI technology giants as Alibaba and google is also under process and creating the latest attempt for digital revolution of health care.

The future of AI-related medical applications also depends on safety, confidentiality and data security. There will be sceptical interest in using algorithms that may risk revealing patient identity in light of hacking, cyber thefts and various instances of data breaches even in the highest cybersecurity zones and legal privacy issues. There are also chances that clinician skill sets and judgments may be weakened from an overdependence on automated AI-based systems with possible increases in medical errors [31].

\section{Conclusion}

The spectrum to which AI technology may change our attitudes to screening and diagnosing thoracic diseases and the overall healthcare scenario is quite massive and full of potential. It is necessary to improve knowledge in this new field and understand the various means by which AI technologies could impact thoracic surgical practice and enhance collaboration with pulmonologists, pathologists and radiologists. Currently, genuinely autonomous surgery by Robots does not exist, and no application seems likely shortly. Nonetheless, taking an interest in recent AI technology with its capabilities and progression in related medical specialities will allow us to improve patient management in various clinical scenarios and future research possibilities. Surgeons can also be strategic in close partnership with other medical specialists and AI engineers, AI designers, and hospital administration to assess AI's relevance and corroborate its usage in their practice to augment better thoracic surgical care. In future, we should not embrace this as 'human versus machine' but rather a cordial partnership to further improve clinical outcomes. This knowledge enhancement is also a fundamental requirement as worldwide governments are increasing their funding in this promising field and changing their legislator conditions to promote AI's safe and secure introduction. The simplest and most recent example is the inclusion and approval of telemedicine use in various countries. With genuine concerns, clinical supervision of AI-based techniques and robotic procedures are an essential prerequisite for further development. However, there are likely to be several other advancements and technologies that may change this scenario in the near future.

\section{Abbreviations \\ Al: Artificial intelligence; ML: Machine learning; DL: Deep learning; ANNs: Artificial neural networks; DNNs: Deep neural networks; \\ CT: Computerised tomography; MRI: Magnetic resonance imaging; Cl: Confidence Interval; MIS: Minimally Invasive Surgery; PARAMIS: Parallel Robot for Minimally Invasive Surgery; CESTER: Centre for Industrial Robots Simulation and Testing; STAR: Smart tissue autonomous robot; USFDA: United States Food and Drug Administration; USD: US Dollar}

\section{Acknowledgements \\ Not Applicable.}

\section{Authors' contributions}

$A D, K K$ and SD initially planned this concept and design. AD, SD and PLP collected and assemble data after researching the literature. Data analysis and interpretation were done by AD, KK and PLP. Manuscript writing was done by all authors. All authors have made substantial contributions and contributed equally in the article's preparation. The requirements for authorship as stated have been met, and that each author believes that the manuscript represents honest work. All authors have read and approved the manuscript. 


\section{Authors' information}

AD is super-specialist cardiothoracic surgeon with a master degree holder in surgery (MS) and later postdoctoral degree in cardio-thoracic surgery branch (M.Ch.). Currently, he is working as an associate professor and Head of Department in All India Institute of Medical science (AllMS) Rishikesh, India, since November 2012. He is the initiator and main thrust behind the development of Heart \& Lung surgery department of the institute in this hilly state of India. This is the first government institute in this remote state to start heart and lung surgery services for deprived patients.

KK is Masters and Post-doctoral degree holder in computer science engineering field with specialisation in Al \& Deep learning fields. Currently, he is an associate professor and Head of computer science engineering (CSE) department of the National Institute of Technology (NIT), Srinagar, Uttarakhand, India.

SD is currently a final year student of the bachelor degree course in computer science engineering field at the Delft University of Technology (TU Delft), Netherlands, with a keen interest in Al \& Deep learning fields. As a multidisciplinary and joint institutional collaboration, all of the above authors are jointly working on the field of the practical application of artificial intelligence in cardio-thoracic surgical field.

\section{Funding}

None

\section{Availability of data and materials}

Not applicable

\section{Declarations}

Ethics approval and consent to participate

Not required - as a review article.

\section{Consent for publication}

Not applicable

\section{Competing interests}

The authors declare that they have no competing interests.

\section{Author details}

'CTVS Department, AllMS, Rishikesh 249203, India. ${ }^{2}$ CSE Department, National Institute of Technology, Srinagar, Uttarakhand 246174, India. ${ }^{3}$ CSE, Technical University, Delft, Netherlands.

\section{Received: 20 April 2021 Accepted: 26 June 2021}

Published online: 03 July 2021

\section{References}

1. Simpkin AL, Schwartzstein RM (2016) Tolerating Uncertainty - The next medical revolution? N Engl J Med 375(18):1713-1715. https://doi.org/10.1 056/NEJMp1606402 PMID: 27806221

2. Jiang $F$, Jiang $Y$, Zhi H, Dong $Y, L i ~ H, M a ~ S$, Wang $Y$, Dong $Q$, Shen $H$, Wang $Y$ (2017) Artificial intelligence in healthcare: past, present and future. Stroke Vasc Neurol 2(4):230-243. https://doi.org/10.1136/svn-2017-000101 PMID: 29507784; PMCID: PMC5829945

3. McCarthy J, Minsky ML, Rochester N, Shannon CE (2006) A proposal for the Dartmouth Summer Research Project on Artificial Intelligence, August 31, 1955. Al Mag 27(4):12. https://doi.org/10.1609/aimag.v27i4.1904

4. Safavi KC, Khaniyev T, Copenhaver M, Seelen M, Zenteno Langle AC, Zanger J, Daily B, Levi R, Dunn P (2019) Development and Validation of a Machine Learning Model to Aid Discharge Processes for Inpatient Surgical Care. JAMA Netw Open 2(12):e1917221. https://doi.org/10.1001/jama networkopen.2019.17221 PMID: 31825503; PMCID: PMC6991195

5. Topol EJ (2019) High-performance medicine: the convergence of human and artificial intelligence. Nat Med 25(1):44-56. https://doi.org/10.1038/s41 591-018-0300-7 Epub 2019 Jan 7. PMID: 30617339

6. Keane PA, Topol EJ (2018) With an eye to Al and autonomous diagnosis. npj Digital Med 1:40. https://doi.org/10.1038/s41746-018-0048-y

7. Kelly CJ, Karthikesalingam A, Suleyman M, Corrado G, King D (2019) Key challenges for delivering clinical impact with artificial intelligence. BMC Med 17(1):195. https://doi.org/10.1186/s12916-019-1426-2
8. Pesapane F, Volonté C, Codari M, Sardanelli F (2018) Artificial intelligence as a medical device in radiology: ethical and regulatory issues in Europe and the United States. Insights Imaging 9(5):745-753. https://doi.org/10.1007/ s13244-018-0645-y Epub 2018 Aug 15. PMID: 30112675; PMCID: PMC6206380

9. Kaul V, Enslin S, Gross SA (2020) History of artificial intelligence in medicine. Gastrointest Endosc 92(4):807-812. https://doi.org/10.1016/.jgie.2020.06.040 Epub 2020 Jun 18. PMID: 32565184

10. Gonem S, Janssens W, Das N, Topalovic M (2020) Applications of artificial intelligence and machine learning in respiratory medicine. Thorax. 75(8): 695-701. https://doi.org/10.1136/thoraxjnl-2020-214556 Epub 2020 May 14. PMID: 32409611

11. Niu H, Gu J (2021) Al driven feature extraction model for chest cavity spectrum signal visualisation. Int J Speech Technol:1-14. https://doi.org/10.1 007/s10772-021-09844-w Epub ahead of print. PMID: 33967593; PMCID: PMC8090519

12. Sakamoto T, Furukawa T, Lami K, Pham HHN, Uegami W, Kuroda K, Kawai M, Sakanashi H, Cooper LAD, Bychkov A, Fukuoka J (2020) A narrative review of digital pathology and artificial intelligence: focusing on lung cancer. Transl Lung Cancer Res 9(5):2255-2276. https://doi.org/10.21037/t|cr-20-591 PMID: 33209648; PMCID: PMC7653145

13. Khemasuwan D, Sorensen JS, Colt HG (2020) Artificial intelligence in pulmonary medicine: computer vision, predictive model and COVID-19. Eur Respir Rev 29(157):200181. https://doi.org/10.1183/16000617.0181-2020 PMID: 33004526; PMCID: PMC7537944

14. Sadeghi AH, Maat APWM, Taverne YJHJ, Cornelissen R, Dingemans A-MC, Bogers AJJC, Mahtab EAF (2021) Virtual reality and artificial intelligence for 3-dimensional planning of lung segmentectomies. JTCVS Tech. https://doi. org/10.1016/j.xjtc.2021.03.016 (ISSN 2666-2507) (https://www.sciencedirect. com/science/article/pii/S2666250721002534)

15. Yang Q, Xie B, Hu M, Sun X, Huang X, Guo M (2016) Thoracoscopic anatomic pulmonary segmentectomy: a 3-dimensional guided imaging system for lung operations. Interact Cardiovasc Thorac Surg 23(2):183-189. https://doi.org/10.1093/icvts/ivw085 Epub 2016 Apr 19. PMID: 27099268

16. Etienne $\mathrm{H}$, Hamdi S, Le Roux M et al (2020) Artificial intelligence in thoracic surgery: past, Present, perspective and limits. Eur Respir Rev 29(157):200010. https://doi.org/10.1183/16000617.0010-2020

17. Gossot D, Abid W, Seguin-Givelet A (2018) Motorized scope positioner for solo thoracoscopic surgery. Video Assist Thorac Surg 3:47. https://doi.org/1 $0.21037 /$ vats.2018.11.01

18. Vaida C, Pisla D, Plitea N, Gherman B, Gyurka B, Stancel E, Hesselbach J, Raatz A, Vlad L, Graur F (2010) Development of a Control System for a Parallel Robot Used in Minimally Invasive Surgery. https:/doi.org/10.1007/ 978-3-642-04292-8_38

19. Pisla D, Plitea N, Vaida C, Hesselbach J, Raatz A, Vlad L, Graur F, Gyurka B, Gherman B, Suciu M. PARAMIS parallel robot for laparoscopic surgery. Chirurgia (Bucur). 2010;105(5):677-83. PMID: 21141094.

20. O'Sullivan S, Nevejans N, Allen C, Blyth A, Leonard S, Pagallo U, Holzinger K, Holzinger A, Sajid Ml, Ashrafian H (2019) Legal, regulatory, and ethical frameworks for development of standards in artificial intelligence (Al) and autonomous robotic surgery. Int J Med Robot 15(1):e1968. https://doi.org/1 0.1002/rcs.1968 PMID: 30397993

21. Zirafa CC, Romano G, Key TH, Davini F, Melfi F (2019) The evolution of robotic thoracic surgery. Ann Cardiothorac Surg 8(2):210-217. https://doi. org/10.21037/acs.2019.03.03 PMID: 31032204; PMCID: PMC6462549

22. Marino MV, Shabat G, Gulotta G, Komorowski AL (2018) From Illusion to Reality: A Brief History of Robotic Surgery. Surg Innov 25(3):291-296. https:// doi.org/10.1177/1553350618771417 Epub 2018 Apr 27. PMID: 29701135

23. Wachter S, Mittelstadt B, Floridi L (2017) Transparent, explainable, and accountable Al for robotics. Sci Robot 2(6):eaan6080. https://doi.org/10.112 6/scirobotics.aan6080 PMID: 33157874

24. Publications Office of the EU (2018) Civil law rules on robotics: European Parliament resolution of 16 February 2017 with recommendations to the Commission on Civil Law Rules on Robotics (2015/2103(INL)). Off J Eur Union:C252/239

25. Wu C, Gleysteen J, Teraphongphom NT, Li Y, Rosenthal E (2018) In-vivo optical imaging in head and neck oncology: basic principles, clinical applications and future directions. Int J Oral Sci 10. https://doi.org/10.1038/ s41368-018-0011-4

26. Navarrete-Welton AJ, Hashimoto DA (2020) Current applications of artificial intelligence for intraoperative decision support in surgery. Front Med 14(4): 
369-381. https://doi.org/10.1007/s11684-020-0784-7 Epub 2020 Jul 3. PMID: 32621201

27. Azhar $H$, Waseem $T$, Ashraf $H$ (2021) Artificial Intelligence in Surgical Education and Training: A Systematic Literature Review. Arch Surg Res 2(1): 58-66. https://doi.org/10.48111/2021.01.10

28. Vellido A (2019) Societal issues concerning the application of artificial intelligence in medicine. Kidney Dis 5(1):11-17. https://doi.org/10.1159/0004 92428

29. Loftus TJ, Filiberto AC, Balch J, Ayzengart AL, Tighe PJ, Rashidi P, Bihorac A, Upchurch GR Jr (2020) Intelligent, Autonomous Machines in Surgery. J Surg Res 253:92-99. https://doi.org/10.1016/j.js.2020.03.046 Epub 2020 Apr 24. PMID: 32339787; PMCID: PMC7594619

30. Gordon L, Grantcharov T, Rudzicz F (2019) Explainable Artificial Intelligence for Safe Intraoperative Decision Support. JAMA Surg 154(11):1064-1065. https://doi.org/10.1001/jamasurg.2019.2821 PMID: 31509185

31. Hwang TJ, Kesselheim AS, Vokinger KN (2019) Lifecycle Regulation of Artificial Intelligence- and Machine Learning-Based Software Devices in Medicine. JAMA. 322(23):2285-2286. https://doi.org/10.1001/jama.2019.1 6842. Epub ahead of print. PMID: 31755907

\section{Publisher's Note}

Springer Nature remains neutral with regard to jurisdictional claims in published maps and institutional affiliations.

\section{Submit your manuscript to a SpringerOpen ${ }^{\circ}$ journal and benefit from:}

- Convenient online submission

- Rigorous peer review

- Open access: articles freely available online

High visibility within the field

- Retaining the copyright to your article

Submit your next manuscript at $\boldsymbol{\nabla}$ springeropen.com 